\title{
Defect analysis of power network equipment based on local evidence
}

\author{
Zhang $\mathrm{Hu}^{1,}$, ,Wang Hongbin ${ }^{1,}$,, Li Feng, a \\ ${ }^{1}$ Electric Power Research Institute of Guangdong Power Grid Co., Ltd., Guangzhou \\ 510000, Guangdong Province, China \\ ànanghu_gd@163.com
}

\begin{abstract}
Keywords: Regional Power Grid; Relay Protection; Online Self-adjusting; Evaluation \& Alarm
\end{abstract}

\begin{abstract}
As the power supply core for power consumers, the regional power grid is responsible for maximally absorbing new energy sources and providing reliable and economic electric energy to users, thus to become an important development link of modern smart power grid. In allusion to actual conditions of regional power grid, the online relay protection setting calculation and the online relay protection evaluation \& pre-alarm are researched in order to solve the problem regarding the relay protection setting value in the regional power grid and the power grid adaptability as well as research and realize the district-county relay protection setting calculation and setting value management integrated system according to the actual condition of the regional power grid in Shijiazhuang City. Such research has important theoretical significance and practical value for improving relay protection performance.
\end{abstract}

\section{Introduction}

As the power grid development tendency in the world, the smart power grid can promote energy conservation and emission reduction and realize low carbon economy, so the construction of smart power grid has become the national strategy in China [1-3]. In consideration of the actual characteristics of energy distribution and national economic development in China, a lot of electric energy needs to be transported from the west to the east. As a result, extra-high voltage remote AC-DC mixed power transmission becomes an important characteristic of the power grids in China, thus making the structure of the power grids in China become more complicated and increasing the difficulty in safe and stable operation. Meanwhile, some renewable energy sources are accessed to low voltage power grids as the distributed generation, thus not only making the power distribution network changed into multi-power system from single power system, but also causing significant and rapid change of power operation mode, more complicated power grid topology, AC-DC mixed transmission and long-short line interconnection. Actually, all these problems have brought severe challenge to relay protection.

As the power supply subject for power consumers, the regional power grid is responsible for absorbing as many as new energy sources and providing reliable and economic electric energy to users, thus to become an important development link of modern smart power grid. Although the regional power grid has relatively low voltage class, compared with high voltage power grid, the regional power grid has many nonstandard connections and multiple relay protection configuration modes as well as certain specificity. The details are as follows:

1) In regional power grid, different lines may have different protection configuration modes and different limiting conditions, so different protection devices need to coordinate with each other. The setting principles of different regional power grids may be different from each other due to different habits of the local relay protection workers. As a result, compared with high voltage power grid, the regional power grid has more complicated relay protection coordination.

2) The complexity of the regional power grid is increased day by day along with the access of many new energy sources to the regional power grid. Meanwhile, the access of the distributed generation to the power distribution network has made the regional power grid changed into multi-power source from single power source, thus significantly changing the operation mode of the 
regional power grid.

As the power supply core for power consumers, the regional power grid is responsible for maximally absorbing new energy sources and providing reliable and economic electric energy to users, thus to become an important development link of modern smart power grid. In allusion to actual conditions of regional power grid, the online relay protection setting calculation and the online relay protection evaluation \& pre-alarm are researched in order to solve the problem regarding the relay protection setting in regional power grid and the power grid adaptability as well as research and realize the district-county relay protection setting calculation and setting value management integrated system according to the actual condition of the regional power grid in Shijiazhuang City. Such research has important theoretical significance and practical value for improving relay protection performance.

\section{Relay Protection System Model for Regional Power Grid}

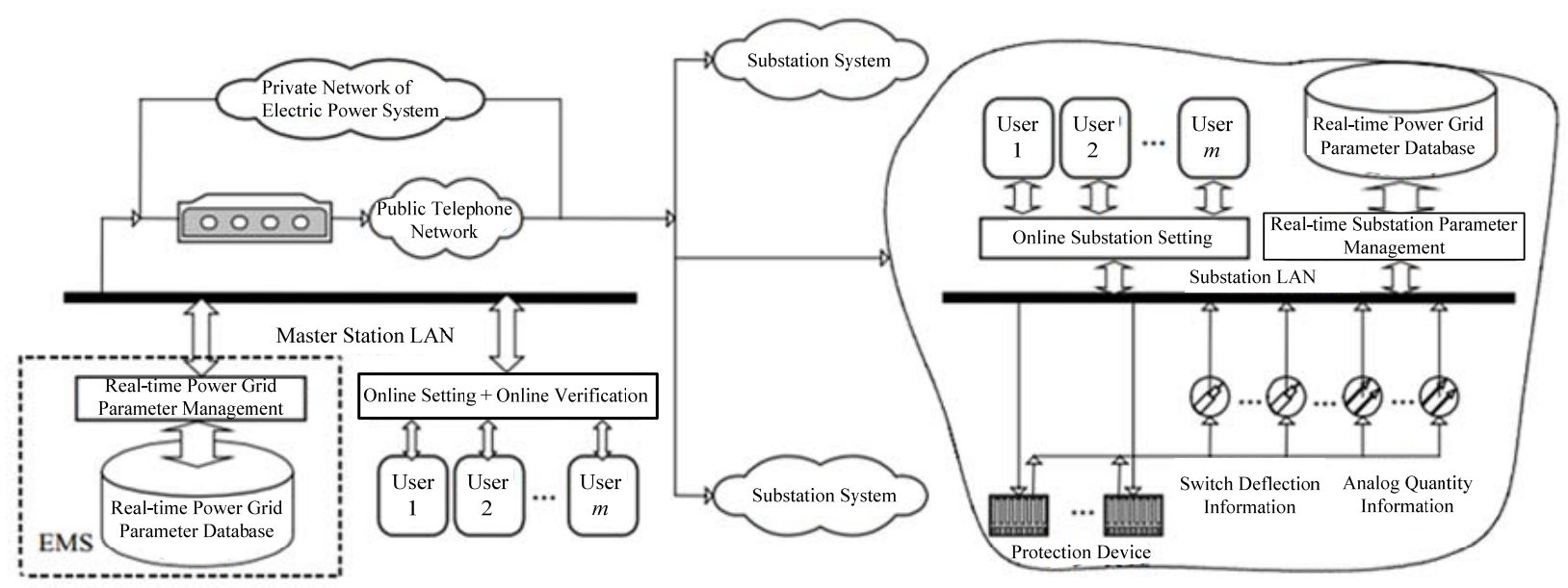

Fig.1. Online relay protection self-adjusting system structure of regional power grid

The online relay protection self-adjusting system structure of the regional power grid system is as shown in Fig.1, and the system includes three major parts, namely: master station system, substation system and protection device, wherein the master station system is placed at the dispatching terminal and the substation system is placed at the substation. The specific functions thereof are as follows:

(1) Protection device

Local setting: according to the position detection information, the protection device judges whether the protection setting value is changed; if it needs to be changed, then the protection device will calculate and directly change the operation protection setting value, wherein the protection setting value is changed according to the remote control information.

(2) Substation system

Substation setting: according to the detection information of various protection devices in the substation and the states of the circuit breakers and the disconnecting switches in the substation, the substation system judges whether the protection setting value in the substation is changed; if it needs to be changed, then the substation system will calculate the protection setting value and send it to various protection devices for changing the operation protection setting value.

(3) Master station system

Centralized setting: according to the information uploaded by all substations, the master station system judges the present system state (direct acquisition of the real-time network state analysis result from the system) in order to realize the centralized protection setting. Afterwards, for the protection setting value to be changed, the master station system will send the new protection setting value to various protection devices through the substation control center. 


\section{Pre-alarm Result Evaluation Criterion}

When the operation mode is changed, the original relay protection setting value will be no longer adaptive or will even cause incorrect operation of relay protection device and accordingly bring potential safety hazard to the power grid. Therefore, it is necessary to quantitatively evaluate the influence of the relay protection setting value irrationality and determine the weak link of the relay protection setting value irrationality in the power grid in order to take corresponding measures to remove faults and hidden dangers. Risk evaluation matrix method is adopted in this article to quantitatively evaluate the influence of the hidden dangers of the relay protection on the power grid security, thus to realize online evaluation \& pre-alarm.

The risk analysis methods include FMEA, event tree, etc., and most risk analysis methods have certain defects. Therein, non-dangerous failure factors rather than various actual comprehensive factors are considered in FMEA method, and this method also causes a waste of time; the event tree not easy to understand usually has multiple mathematical solutions and includes complicated logical relation. For comprehensive consideration, the risk evaluation matrix method is adopted in order to make the risk analysis result more intuitional and accurate. The verification rule for the protection setting value $(110 \mathrm{kV})$ is as shown in Table 1.

Tab.1. Verification rule for protection setting value $(110 \mathrm{kV})$

\begin{tabular}{|c|c|c|c|c|}
\hline $\begin{array}{l}\text { Voltage } \\
\text { Class }\end{array}$ & Type & $\begin{array}{l}\text { Section } \\
\text { No. }\end{array}$ & Sensitivity Planning & Selectivity Planning \\
\hline \multirow{6}{*}{$110 \mathrm{KV}$} & \multirow{3}{*}{$\begin{array}{l}\text { Zero } \\
\text { Sequence } \\
\text { Current } \\
\text { Protection }\end{array}$} & Section I & & $\begin{array}{l}\text { With reliable coefficient for } \\
\text { the external maximum fault } \\
\text { zero sequence current }\end{array}$ \\
\hline & & Section II & $\begin{array}{l}\text { Line less than } 20 \mathrm{~km}: \geq 1.5 \\
\text { Line between } 20 \mathrm{~km} \text { and } \\
50 \mathrm{~km}: \geq 1.4 \text {; } \\
\text { Line more than } 50 \mathrm{~km}: \geq 1.3\end{array}$ & $\begin{array}{l}\text { Within the temporal next } \\
\text { protection range; within the } \\
\text { protection range of the high } \\
\text { voltage bus of } 220 \mathrm{KV} \\
\text { transformer }\end{array}$ \\
\hline & & Section III & Adjacent bound lines: $\geq 1.2$ & $\begin{array}{l}\text { Within the temporal next } \\
\text { protection range; without } \\
\text { exceeding the zero sequence } \\
\text { current under the high } \\
\text { voltage side fault of } 220 \mathrm{KV} \\
\text { transformer }\end{array}$ \\
\hline & \multirow{3}{*}{$\begin{array}{c}\text { Equidistance } \\
\text { Protection }\end{array}$} & Section I & & $\begin{array}{l}\text { Bypassing the same fault of } \\
\text { bound lines; within the } \\
\text { protection range of the bus at } \\
\text { other sides of the transformer } \\
\text { at the line terminal }\end{array}$ \\
\hline & & Section II & $\begin{array}{l}\text { Line less than } 20 \mathrm{~km}: \geq 1.5 ; \\
\text { Line between } 20 \mathrm{~km} \text { and } \\
50 \mathrm{~km}: \geq 1.4 ; \\
\text { Line more than } 50 \mathrm{~km}: \geq 1.3 ;\end{array}$ & $\begin{array}{l}\text { Within the temporal next } \\
\text { protection range; within the } \\
\text { protection range of the bus at } \\
\text { other sides of the transformer }\end{array}$ \\
\hline & & Section III & Adjacent bound lines: $\geq 1.2$ & $\begin{array}{l}\text { Within the temporal next } \\
\text { protection range; bypassing } \\
\text { the minimum accident } \\
\text { overload impedance of the } \\
\text { line }\end{array}$ \\
\hline
\end{tabular}




\begin{tabular}{c|c|c|l|l}
\hline $\begin{array}{c}\text { Voltage } \\
\text { Class }\end{array}$ & Type & $\begin{array}{c}\text { Section } \\
\text { No. }\end{array}$ & \multicolumn{1}{|c|}{ Sensitivity Planning } & \multicolumn{1}{c}{ Selectivity Planning } \\
\hline Section I & $\begin{array}{c}\text { Equidistance } \\
\text { Protection }\end{array}$ & Section II & $\begin{array}{l}\text { Line between } 20 \mathrm{~km} \quad \text { and } \\
50 \mathrm{~km}: \geq 1.4 ; \\
\text { Line more than } 50 \mathrm{~km}: \geq 1.3 ; \\
\text { bound lines; within the bus at } \\
\text { other sides of the transformer } \\
\text { at the line terminal }\end{array}$ \\
\cline { 3 - 6 } & Section III & $\begin{array}{l}\text { Within the temporal next } \\
\text { protection range; within the } \\
\text { protection range of the bus at } \\
\text { other sides of the transformer }\end{array}$ \\
\hline
\end{tabular}

\section{Setting System Workflow}

The protocol used for the communication between fault information master station and substations is inapplicable to the functions of protection setting value change and file downloading, so the protocol definition is expanded during the system research and development on original basis. The remote online automatic device value setting system constructed on the fault information master station can acquire the main content of XML setting value form and then compare it with the internal device setting value in order to display the inconsistent setting value and meanwhile remotely online change the device setting value. If the user needs to locally and automatically set the device setting value, the user can download XML setting value form to the substation through the master station and then adopt the debugging software of the device manufacturer to locally and automatically set the device setting value.

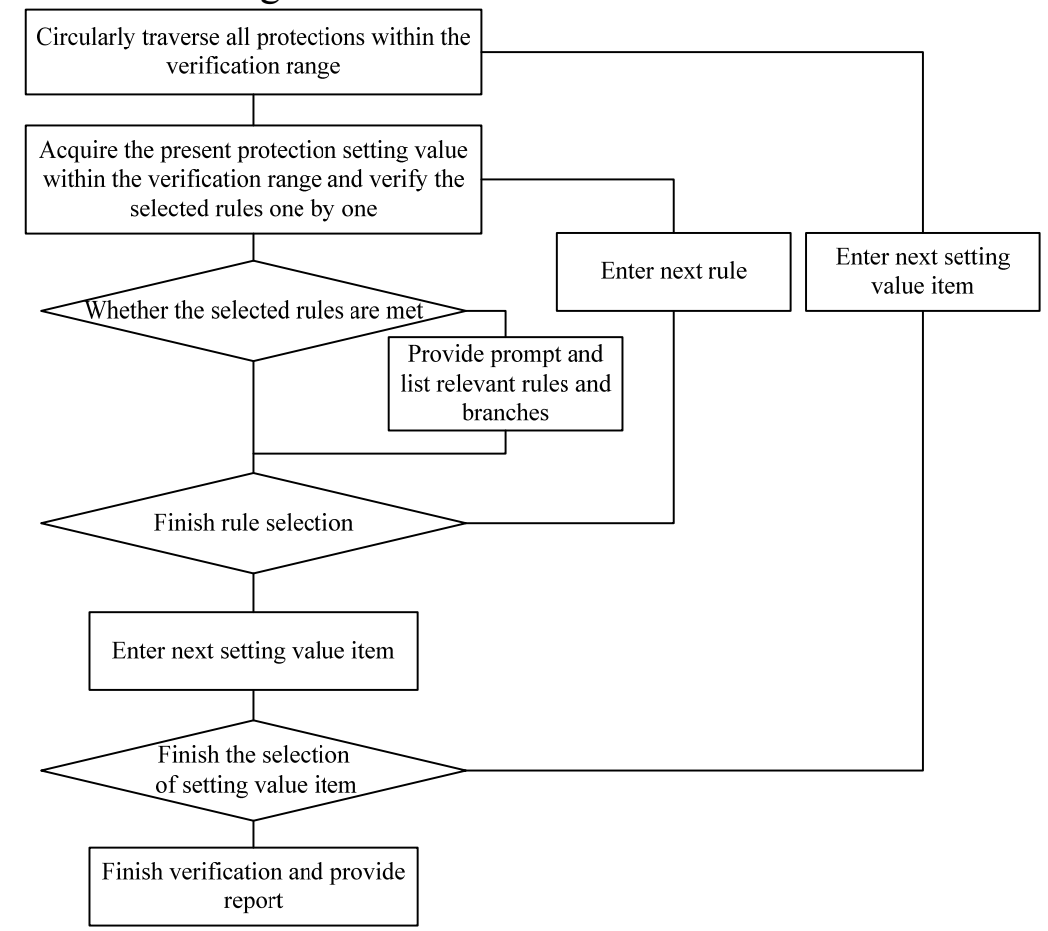

Fig.2. Process flow of automatic setting value verification function

The process flow of the automatic setting value verification function is as shown in Fig.2, and the details are as follows:

1) Validity check of XML setting value form: firstly, orderly extract the setting value content in XML setting value form and place such content in a structural buffer, then use SAX to analyze XML file, and then compare the template description in present buffer with the called device version and type. In case of consistency, enter the setting value conversion part; or else, the system will report error. 
2) Setting value conversion verification: convert various types (hexadecimal, integer, floating point, etc.) of setting values into the numerical value able to be distinguished by the corresponding device; compare the validity of the setting values and check whether each setting value is within the designated range; in case of any over-range behavior, give the comparison result after comparison.

3) Device setting value downloading: after comparing the setting value in XML setting value form with the device setting value, display the inconsistent item; after user confirmation, take relevant protocol for the setting value transmission, wherein the master station only needs to download the setting value to be changed and the substation shall be responsible for changing the device setting value, and for local automatic setting, such work is finished by the debugging software of the manufacturer; After setting value change, call the changed setting value and compare it with the setting value in the buffer; if confirmation is returned, it is indicated that the automatic setting of the device setting value is successfully finished.

\section{Realization of Online Relay Protection Setting}

Along with the improvement of the requirements for power grid accident analysis and processing, the fault information system plays a more and more important role in the dispatching automation system. In consideration of different timeliness and integration requirements for different fault information systems when the power grid suffers from faults, this system hierarchically processes the information and provides effective assistance for fault analysis and processing for the power grid through the interaction of such information as failure logging, fault recording, fault analysis and fault handling result finished by the information master station and substation.

On the basis of combining the special setting value unit or fault information system, the online self-adjusting system communicates with the protection devices at the station terminal through the fault information channel in order to realize the online protection value setting function for the system, and the basic structure of the system is as shown in Fig.3.

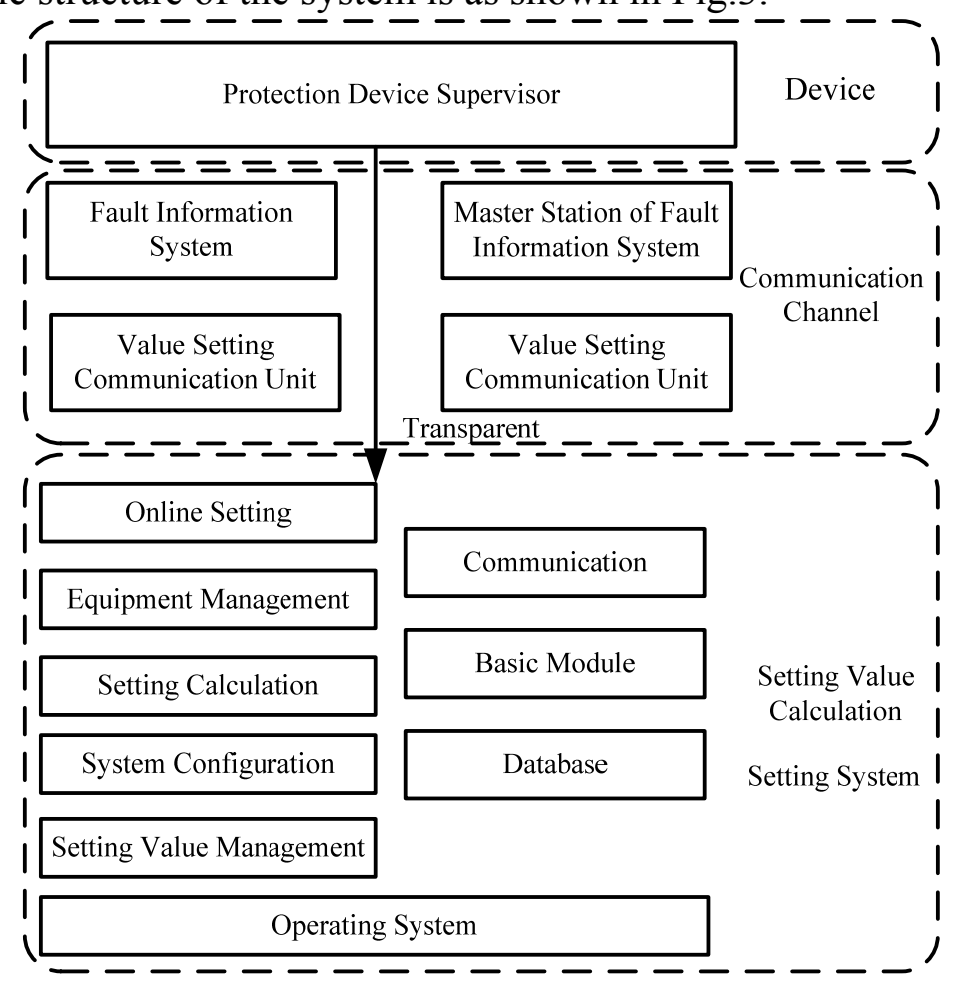

Fig.3. Functional block diagram of online setting system

Online setting can not only avoid the adverse influence of operation uncertainty on protection setting value performance, but also obviously improve protection performance. The online setting value and the original offline setting value of a certain regional power grid are statistically compared, and the result is as shown in Fig.4. In the figure: the vertical white band denotes the online setting result and the vertical black band denotes the offline setting result. Obviously, 
compared with the offline setting value, the online setting value has high sensitivity, less mismatched protections and short time delay.
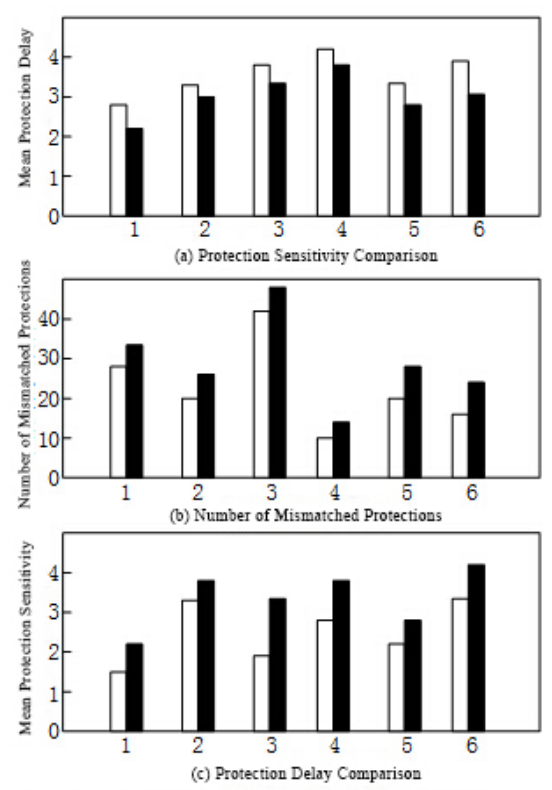

1-Section II of Zero Sequence Current 2-Section III of Zero Sequence Curren

3-Section II of Grounding Distance 4 -Section III of Grounding Distance

5-Section II of Phase Spacing 6-Section III of Phase Spacing

Fig.4. Comparison between online setting and offline setting

\section{Conclusion}

In order to meet the requirements regarding the four characteristics (reliability, selectivity, rapidness and sensitivity) of the relay protection for the power grid and give full play to the efficiency of the relay protection device, this article focuses on researching the key technology for online relay protection setting and evaluation \& pre-alarm, and the following conclusions are obtained: 1) the accurate fault calculation model is adopted for the online protection value setting system mentioned in this article and the setting value is determined point by point along the line in order to avoid the calculation error brought by the introduction of branch coefficient into the offline setting. 2) The influence of the local change of the power grid on the protection setting value and the actual calculation instance are also analyzed in order to explain that various local changes of the power grid only locally influences the protection setting value. The online self-adjusting technology can improve the action speed and accuracy of the relay protection system.

\section{References}

[1] Zhang, Mengxin, Zhihan Lv, Xiaolei Zhang, Ge Chen, and Ke Zhang. "Research and Application of the 3D Virtual Community Based on WEBVR and RIA." Computer and Information Science 2, no. 1 (2009): p84.

[2] Su, Tianyun, Zhihan Lv, Shan Gao, Xiaolong Li, and Haibin Lv. "3D seabed: 3D modeling and visualization platform for the seabed." In Multimedia and Expo Workshops (ICMEW), 2014 IEEE International Conference on, pp. 1-6. IEEE, 2014.

[3] Jiang, Dingde, Zhengzheng Xu, Peng Zhang, and Ting Zhu. "A transform domain-based anomaly detection approach to network-wide traffic." Journal of Network and Computer Applications 40 (2014): 292-306.

[4] J. He, Y. Geng and K. Pahlavan, Modeling Indoor TOA Ranging Error for Body Mounted Sensors, 2012 IEEE 23nd International Symposium on Personal Indoor and Mobile Radio Communications (PIMRC), Sydney, Australia Sep. 2012 (page 682-686). 